\title{
Analysis of Transfer Line Systems with a Merge Stage for Three Kinds of Parts at Discrete Times
}

\author{
Mitsuo Yamashiro, Takayuki Takagi and Yorimasa Kuba
}

\author{
Department of Industrial and Information Systems Engineering, Ashikaga Institute of Technology \\ 268-1, Ohmae-cho, Ashikaga, 326-8558 Japan \\ Phone: (+81)284-62-0605 \\ Fax: (+81)284-64-1071 \\ E-mail : yamasiro@ashitech.ac.jp,g01501@ashitech.ac.jp, quba@ashitech.ac.jp
}

\begin{abstract}
In this study, we analyzed two merge-type transfer line systems by the Markov process at discrete times. The first system is the merge-type transfer line system of three parallel processes. That is, it is a manufacturing process in which three kinds of parts, $A, B$ and $C$, are assembled in parallel and are completed at the same time. Next, the second system is the merge-type transfer line system with two stages in which three kinds of parts completed in the first stage are supplied to the next stage in series. Examples of numerical calculations were given for the obtained results.
\end{abstract}

\section{Introduction}

The process design problem is the flow of the work about the production process that changes material into the product. The decisions are made about the flow of materials. In this design, emphasis is put on the smoothness of the workflow, which mainly depends on the degree of skill of the material handling. Three kinds of the workflow patterns are considered [1]. The 1 st pattern is the straight line process, which arranges several processes in series. The 2nd pattern is the divergent process that branched off into plural parallel process, and plural product is made when the processes progress. The 3rd pattern is the merge-type process. It made by combining into a single process from several parallel processes.

In this study, the merge-type transfer line system of the 3 rd pattern and the series-type transfer line system of the $1 \mathrm{st}$ pattern are treated $[2,3]$. The first system is the merge-type transfer line system of three parallel processes. This is a process in which three kinds of parts, $A, B$ and $C$ are assembled in parallel, and the three parts of $A, B$ and $C$ are completed at the same time. The second system is the merge-type transfer line system with two stages in which three kinds of parts completed in the first process are supplied to the following process in the series. In this study, we analyze the two transfer line systems by the Markov process at discrete times [4-7]. Markov process at discrete time is well known as one of useful methods for analyzing the system with stochastic behaviors. Further, when parameters of the process are changed, it is shown by numerical examples how many the quantity of the part stock is left. When cost in the system is decreased, it is important to reduce the part stock.

\section{Analysis}

\section{1 Merge-type Transfer Line System}

Three kinds of parts $A, B$ and $C$ are made, and we consider the merge-type transfer line system of three parallel processes that supply the following assembly process with the three parts, $A, B$ and $C$. The three parts are completed at the same time (Fig. 1). $p_{1}$ is the probability that a part $A$ is completed at the process $1.1-p_{1}$ is the probability that a part $A$ is not completed at the process 1 . $p_{2}$, is the probability that a part $B$ is completed at the process $2.1-p_{2}$ is the probability that a part $B$ is not completed at the process 2 . Similarly, $p_{3}$ is the probability that a part $C$ is completed at the process $3.1-p_{3}$ is the probability that a part $C$ is not completed at the process 3 . As for processes 1,2 and 3, the sizes of stocks of the completed parts are limited to $K_{1}, K_{2}$ and $K_{3}$, respectively. When the stock of the completed part becomes full, the process production stops. When three parts are completed at the same time, they are immediately supplied to the following processes. Therefore, for the stocks of the parts, $A$, $B$ and $C$, one is always zero at least.

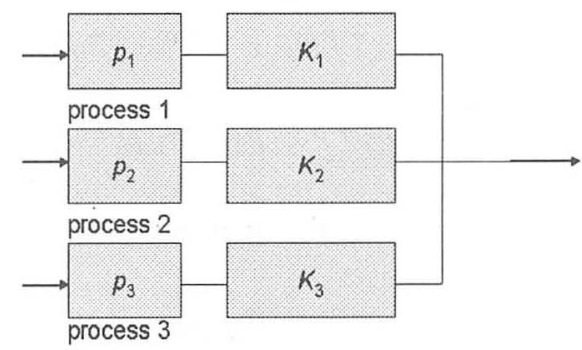

Fig. 1 Merge-type transfer line system

It simplifies the problem that $K_{1}, K_{2}$ and $K_{3}$ in the system are generalized. We put $K_{1}=K_{2}=K_{3}=K$. We treat the case of $K=1$. The state of the system is denoted as the stock numbers $(i, j, m)$ of part $A$, part $B$ and part $C$. The possible state of the system in case of $K=1$ becomes the seven states of $(0,0,0),(1,0,0),(0,1,0),(0,0,1),(1,1,0),(1,0,1)$ and $(0,1,1)$. Here, we put $w_{(i, j, m)}(k)$ as the probability which is the state $(i, j, m)$ of the system at time $k$. The transition from time $k$ to time $k+1$ is considered under the assumptions described above. The state equations in the system are derived as follows. 


$$
\begin{array}{r}
w_{(0,0,0)}(k+1)=p_{1} p_{2} p_{3} w_{(0,0,0)}(k)+\left(1-p_{1}\right)\left(1-p_{2}\right)\left(1-p_{3}\right) w_{(0,0,0)}(k) \\
+p_{2} p_{3} w_{(1,0,0)}(k)+p_{1} p_{3} w_{(0,1,0)}(k)+p_{1} p_{2} w_{(0,0,1)}(k) \\
+p_{3} w_{(1,1,0)}(k)+p_{2} w_{(1,0,1)}(k)+p_{1} w_{(0,1,1)}(k)
\end{array}
$$

$w_{(1,0,0)}(k+1)=p_{1}\left(1-p_{2}\right)\left(1-p_{3}\right) w_{(0,0,0)}(k)+\left(1-p_{2}\right)\left(1-p_{3}\right) w_{(1,0,0)}(k)$

$w_{(0,1,0)}(k+1)=p_{2}\left(1-p_{1}\right)\left(1-p_{3}\right) w_{(0,0,0)}(k)+\left(1-p_{1}\right)\left(1-p_{3}\right) w_{(0,1,0)}(k)$

$w_{(0,0,1)}(k+1)=p_{3}\left(1-p_{1}\right)\left(1-p_{2}\right) w_{(0,0,0)}(k)+\left(1-p_{1}\right)\left(1-p_{2}\right) w_{(0,0,1)}(k)$

$$
\begin{aligned}
w_{(1,1,0)}(k+1)=p_{1} p_{2}(1 & \left.-p_{3}\right) w_{(0,0,0)}(k)+p_{2}\left(1-p_{3}\right) w_{(1,0,0)}(k) \\
& +p_{1}\left(1-p_{3}\right) w_{(0,1,0)}(k)+\left(1-p_{3}\right) w_{(1,1,0)}(k)
\end{aligned}
$$

$$
\begin{aligned}
w_{(1,0,1)}(k+1)=p_{1} p_{3}(1 & \left.-p_{2}\right) w_{(0,0,0)}(k)+p_{3}\left(1-p_{2}\right) w_{(1,0,0)}(k) \\
& +p_{1}\left(1-p_{2}\right) w_{(0,0,1)}(k)+\left(1-p_{2}\right) w_{(1,0,1)}(k)
\end{aligned}
$$

$$
\begin{aligned}
w_{(0,1,1)}(k+1)=p_{2} p_{3}\left(1-p_{1}\right) w_{(0,0,0)}(k)+p_{3}\left(1-p_{1}\right) w_{(0,1,0)}(k) \\
+p_{2}\left(1-p_{1}\right) w_{(0,0,1)}(k)+\left(1-p_{1}\right) w_{(0,1,1)}(k)
\end{aligned}
$$

For the state of the system, the following steady state probability is assumed.

$$
\lim _{k \rightarrow \infty} w_{(i, j, m)}(k)=w_{(i, j, m)}
$$

In this study, when the system in the steady state is treated, after arranging eq.(1) eq.(7) using eq.(8), the state equations are derived as follows.

$$
\begin{aligned}
& -\left(p_{1}+p_{2}+p_{3}-p_{1} p_{2}-p_{1} p_{3}-p_{2} p_{3}\right) w_{(0,0,0)}+p_{2} p_{3} w_{(1,0,0)} \\
& \quad+p_{1} p_{3} w_{(0,1,0)}+p_{1} p_{2} w_{(0,0,1)}+p_{1} w_{(0,1,1)}+p_{2} w_{(1,0,1)}+p_{3} w_{(1,1,0)}=0 \\
& p_{1}\left(1-p_{2}\right)\left(1-p_{3}\right) w_{(0,0,0)}-\left(p_{2}+p_{3}-p_{2} p_{3}\right) w_{(1,0,0)}=0 \\
& p_{2}\left(1-p_{1}\right)\left(1-p_{3}\right) w_{(0,0,0)}-\left(p_{1}+p_{3}-p_{1} p_{3}\right) w_{(0,1,0)}=0 \\
& p_{3}\left(1-p_{1}\right)\left(1-p_{2}\right) w_{(0,0,0)}-\left(p_{1}+p_{2}-p_{1} p_{2}\right) w_{(0,0,1)}=0 \\
& p_{1} p_{2}\left(1-p_{3}\right) w_{(0,0,0)}+p_{2}\left(1-p_{3}\right) w_{(1,0,0)} \\
& \quad+p_{1}\left(1-p_{3}\right) w_{(0,1,0)}-p_{3} w_{(1,1,0)}=0 \\
& p_{1} p_{3}\left(1-p_{2}\right) w_{(0,0,0)}+p_{3}\left(1-p_{2}\right) w_{(1,0,0)} \\
& \quad+p_{1}\left(1-p_{2}\right) w_{(0,0,1)}-p_{2} w_{(1,0,1)}=0 \\
& \quad+p_{2}\left(1-p_{1}\right) w_{(0,0,1)}-p_{1} w_{(0,1,1)}=0
\end{aligned}
$$

The condition of the sum of probabilities becomes 1 .

$$
w_{(0,0,0)}+w_{(1,0,0)}+w_{(0,1,0)}+w_{(0,0,1)}+w_{(1,1,0)}+w_{(1,0,1)}+w_{(0,1,1)}=1
$$

Solving the simultaneous eq.(9) eq.(16), the state probabilities are obtained. Next, the mean number of the part stock, $L_{1}$ as a system characteristic quantity can be obtained using these state probabilities.

$$
L_{1}=w_{(1,0,0)}+w_{(0,1,0)}+w_{(0,0,1)}+2\left(w_{(1,1,0)}+w_{(1,0,1)}+w_{(0,1,1)}\right)
$$

\section{2 Merge-type Transfer Line System with Two Stages}

When three kinds of parts $A$ (process 1), $B$ (process 2) and $C$ (process 3 ) completed in the first stage, we consider the merge-type transfer line system with two stages. The system is conveyed to the following stage (process 4 ) in series. The system has three kinds of parts $A, B$ and $C$, which are supplied the next stage when the parts $A, B$ and $C$ are completed in parallel and are gathered at the same time (Fig.2).

Here, $p_{1}$ is the probability that a part $A$ is completed at the process $1.1-p_{1}$ is the probability that a part $A$ is not completed at the process $1 . p_{2}$ is the probability that a part $B$ is completed at the process $2.1-p_{2}$ is the probability that a part $B$ is not completed at the process 2 . Similarly, $p_{3}$ is the probability that a part $C$ is completed at the process 3.1 $-p_{3}$ is the probability that a part $C$ is completed at the process 3. As for processes 1, 2 and 3, the sizes of stocks of the completed parts are limited to $K_{1}, K_{2}$ and $K_{3}$, respectively. When the stock of the completed part becomes full, the production in the process stops. When three parts are completed at the same time, they are immediately supplied to the following process 4 . For process $4, q$ is the probability that the parts $A, B$ and $C$ are assembled into the product.

As for this section, like the section 2.1, the stocks of the completed parts in processes 1,2 and 3 are limited by $K_{1}$, $K_{2}$ and $K_{3}$, respectively. Similarly, the stocks of the completed parts are limited to $K_{4}$ before process 4 .

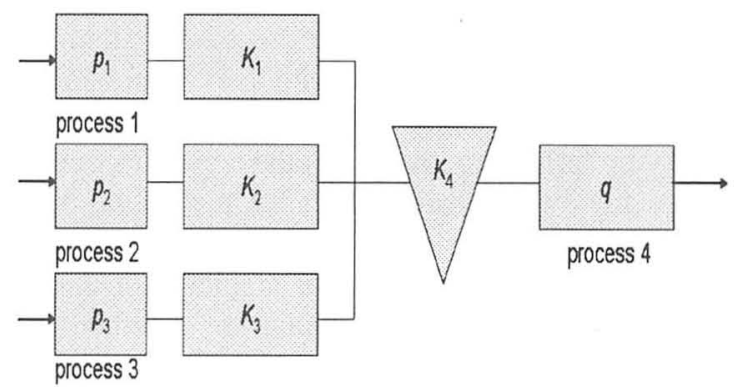

Fig. 2 Merge-type transfer line system with two stages

It simplifies the problem that $K_{1}, K_{2}, K_{3}$ and $K_{4}$ in the system are generalized. We put $K_{1}=K_{2}=K_{3}=K$ and $K_{4}=0$. We treat the case of $K=1$. The state of the system is denoted as the stock numbers $(i, j, m)$ of part $A$, part $B$ and part $C$. The possible state of the system in case of $K=1$ becomes the eight states of $(0,0,0),(1,0,0),(0,1,0),(0,0,1),(1,1,0)$, $(1,0,1),(0,1,1)$ and $(1,1,1)$. Here, we put $w_{(i, j, m)}(k)$ as 
the probability which is the state $(i, j, m)$ of the system at time $k$. The transition from time $k$ to time $k+1$ is considered under the assumptions described above. The state equations in the system are derived as follows. $w_{(0,0,0)}(k+1)=p_{1} p_{2} p_{3} q w_{(0,0,0)}(k)+\left(1-p_{1}\right)\left(1-p_{2}\right)\left(1-p_{3}\right) w_{(0,0,0)}(k)$

$$
\begin{gathered}
+p_{2} p_{3} q w_{(1,0,0)}(k)+p_{1} p_{3} q w_{(0,1,0)}(k)+p_{1} p_{2} q w_{(0,0,1)}(k) \\
+p_{3} q w_{(1,1,0)}(k)+p_{2} q w_{(1,0,1)}(k)+p_{1} q w_{(0,1,1)}(k)+q w_{(1,1,1)}(k)
\end{gathered}
$$

$$
w_{(1,0,0)}(k+1)=p_{1}\left(1-p_{2}\right)\left(1-p_{3}\right) w_{(0,0,0)}(k)+\left(1-p_{2}\right)\left(1-p_{3}\right) w_{(1,0,0)}(k)
$$

$$
w_{(0,1,0)}(k+1)=p_{2}\left(1-p_{1}\right)\left(1-p_{3}\right) w_{(0,0,0)}(k)+\left(1-p_{1}\right)\left(1-p_{3}\right) w_{(0,1,0)}(k)
$$

$$
w_{(0,0,1)}(k+1)=p_{3}\left(1-p_{1}\right)\left(1-p_{2}\right) w_{(0,0,0)}(k)+\left(1-p_{1}\right)\left(1-p_{2}\right) w_{(0,0,1)}(k)
$$

$$
\begin{aligned}
w_{(1,1,0)}(k+1)=p_{1} p_{2}(1 & \left.-p_{3}\right) w_{(0,0,0)}(k)+p_{2}\left(1-p_{3}\right) w_{(1,0,0)}(k) \\
& +p_{1}\left(1-p_{3}\right) w_{(0,1,0)}(k)+\left(1-p_{3}\right) w_{(1,1,0)}(k)
\end{aligned}
$$

$$
\begin{aligned}
w_{(1,0,1)}(k+1)=p_{1} p_{3}(1 & \left.-p_{2}\right) w_{(0,0,0)}(k)+p_{3}\left(1-p_{2}\right) w_{(1,0,0)}(k) \\
& +p_{1}\left(1-p_{2}\right) w_{(0,0,1)}(k)+\left(1-p_{2}\right) w_{(1,0,1)}(k)
\end{aligned}
$$

$$
\begin{aligned}
w_{(0,1,1)}(k+1)=p_{2} p_{3}\left(1-p_{1}\right) w_{(0,0,0)}(k)+p_{3}\left(1-p_{1}\right) w_{(0,1,0)}(k) \\
+p_{2}\left(1-p_{1}\right) w_{(0,0,1)}(k)+\left(1-p_{1}\right) w_{(0,1,1)}(k)
\end{aligned}
$$

$$
\begin{aligned}
w_{(1,1,1)}(k+1)= & p_{1} p_{2} p_{3}(1-q) w_{(0,0,0)}(k)+p_{2} p_{3}(1-q) w_{(1,0,0)}(k) \\
& +p_{1} p_{3}(1-q) w_{(0,1,0)}(k)+p_{1} p_{2}(1-q) w_{(0,0,1)}(k) \\
& +p_{3}(1-q) w_{(1,1,0)}(k)+p_{2}(1-q) w_{(1,0,1)}(k) \\
& +p_{1}(1-q) w_{(0,1,1)}(k)+(1-q) w_{(1,1,1)}(k)
\end{aligned}
$$

For the state of the system, the following steady state probability is assumed.

$\lim _{k \rightarrow \infty} w_{(i, j, m)}(k)=w_{(i, j, m)}$

In this study, when the system in the steady state is treated, after arranging eq.(18) eq.(25) using eq.(26), the state equations are derived as follows.

$$
\begin{array}{r}
p_{1} p_{2} p_{3} q w_{(0,0,0)}-\left(p_{1}+p_{2}+p_{3}-p_{1} p_{2}-p_{1} p_{3}-p_{2} p_{3}+p_{1} p_{2} p_{3}\right) w_{(0,0,0)} \\
+p_{2} p_{3} q w_{(1,0.0)}+p_{1} p_{3} q w_{(0,1,0)}+p_{1} p_{2} q w_{(0,0,1)}+p_{3} q w_{(1,1,0)} \\
+p_{2} q w_{(1,0,1)}+p_{1} q w_{(0,1,1)}+q w_{(1,1,1)}=0
\end{array}
$$

$p_{1}\left(1-p_{2}\right)\left(1-p_{3}\right) w_{(0,0,0)}-\left(p_{2}+p_{3}-p_{2} p_{3}\right) w_{(1,0,0)}=0$

$$
\begin{gathered}
p_{2}\left(1-p_{1}\right)\left(1-\bar{p}_{3}\right) w_{(0,0,0)}-\left(p_{1}+p_{3}-p_{1} p_{3}\right) w_{(0,1,0)}=0 \\
p_{3}\left(1-p_{1}\right)\left(1-p_{2}\right) w_{(0,0,0)}-\left(p_{1}+p_{2}-p_{1} p_{2}\right) w_{(0,0,1)}=0 \\
p_{1} p_{2}\left(1-p_{3}\right) w_{(0,0) 0}+p_{2}\left(1-p_{3}\right) w_{(1,0)}+p_{1}\left(1-p_{3}\right) w_{(0,1,0)}-p_{3} w_{(1,1,0)}=0 \\
p_{1} p_{3}\left(1-p_{2}\right) w_{(0,0)}+p_{3}\left(1-p_{2}\right) w_{(1,0,0)}+p_{1}\left(1-p_{2}\right) w_{(0,0,1)}-p_{2} w_{(1,0,1)}=0 \\
p_{2} p_{3}\left(1-p_{1}\right) w_{(0,0,0)}+p_{3}\left(1-p_{1}\right) w_{(0,1,0)}+p_{2}\left(1-p_{1}\right) w_{(0,0,1)}-p_{1} w_{(0,1)}=0 \\
p_{1} p_{2} p_{3}(1-q) w_{(0,0,0)}+p_{2} p_{3}(1-q) w_{(1,0,0)}+p_{1} p_{3}(1-q) w_{(0,1,0)} \\
\quad+p_{1} p_{2}(1-q) w_{(0,0,1)}+p_{3}(1-q) w_{(1,1,0)}+p_{2}(1-q) w_{(1,0,1)} \\
\quad+p_{1}(1-q) w_{(0,1,1)}-q w_{(1,1,1)}=0
\end{gathered}
$$

The condition of the sum of probabilities becomes 1 .

$$
\begin{aligned}
& w_{(0,0,0)}+w_{(1,0,0)}+w_{(0,1,0)}+w_{(0,0,1)}+w_{(1,1,0)}+w_{(1,0,1)}+w_{(0,1,1)} \\
& +w_{(1,1,1)}=1
\end{aligned}
$$

Solving the simultaneous eq.(25) eq.(35), the state probabilities are obtained. Next, $L_{2}$ is the mean number of the part stock, as a system characteristic quantity can be obtained using these state probabilities.

$$
L_{2}=w_{(1,0,0)}+w_{(0,1,0)}+w_{(0,0,1)}+2\left(w_{(1,1,0)}+w_{(1,0,1)}+w_{(0,1,1)}\right)+3 w_{(1,1,1)}
$$

\section{Examples of Numerical Calculation}

In the first example, we use $K_{1}=K_{2}=K_{3}=1$ in the merge-type transfer line system with three parallel processes of section 2.1. When we put $p_{1}=0.8, p_{2}=0.7$, $p_{3}=0.6$, the steady state probabilities and the mean number of the part stock, $L_{1}$, can be evaluated by solving eq.(9) eq.(16). The computational results are showed as follows;

$$
\begin{aligned}
& w_{(0,0,0)}=0.49196, w_{(1,0,0)}=0.05367, \\
& w_{(0,1,0)}=0.02995, w_{(0,0,1)}=0.01884, \\
& w_{(1,1,0)}=0.22468, w_{(1,0,1)}=0.12146, \\
& w_{(0,1,1)}=0.05945, L_{1}=0.91363 .
\end{aligned}
$$

Next, Fig. 3 shows the relation between $p_{1}$ and $L_{1}$ in the case when $p_{1}=0.1 \sim 0.9, p_{2}=0.7, p_{3}=0.6$. This figure shows that $L_{1}$ decreases when $p_{1}$ becomes higher. 


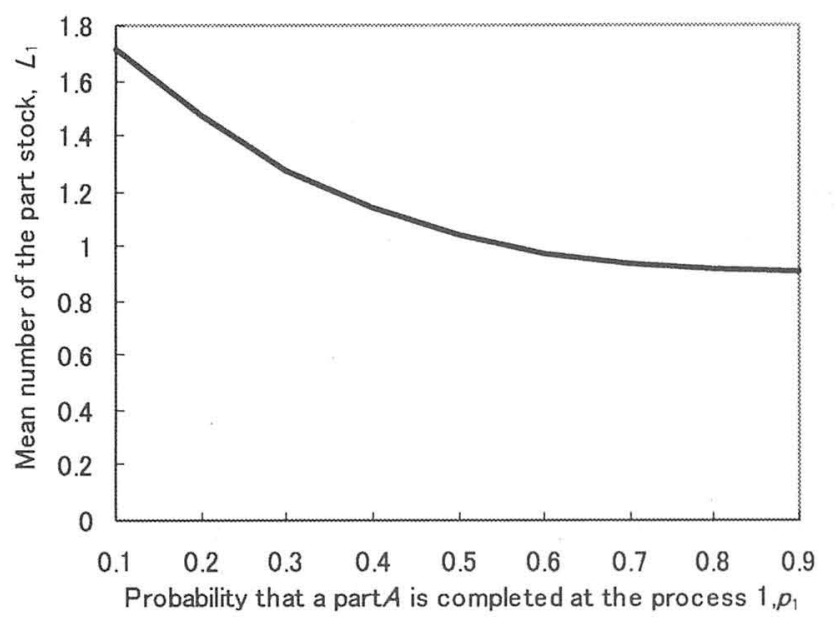

Fig. $3 p_{1}$ vs. $L_{1}\left(p_{2}=0.7, p_{3}=0.6\right)$

In the second example, we use $K_{1}=K_{2}=K_{3}=1, K_{4}=0$ in the merge-type transfer line system with two stages of section 2.2. When we put $p_{1}=0.8, p_{2}=0.7, p_{3}=0.6, q=0.5$ to the steady state probabilities and put $L_{2}$ to the mean number of the part stock, the state equations can be evaluated by solving eq. (27) eq.(35). The computation results are showed as follows:

$$
\begin{aligned}
& w_{(0.0,0)}=0.33237, w_{(1,0,0)}=0.03626, \\
& w_{(0,1,0)}=0.02023, w_{(0,0,1)}=0.01273, \\
& w_{(1,1,0)}=0.15180, w_{(1,0,1)}=0.08206, \\
& w_{(0.1,1)}=0.04016, w_{(1,1,1)}=0.32439, \\
& L_{2}=1.59043 .
\end{aligned}
$$

Next, Fig. 4 shows the relation between $q$ and $L_{2}$ in the case when $p_{1}=0.8, p_{2}=0.7, p_{3}=0.6, q=0.1 \sim 0.9$. This figure shows that $L_{2}$ decreases when $q$ becomes higher.

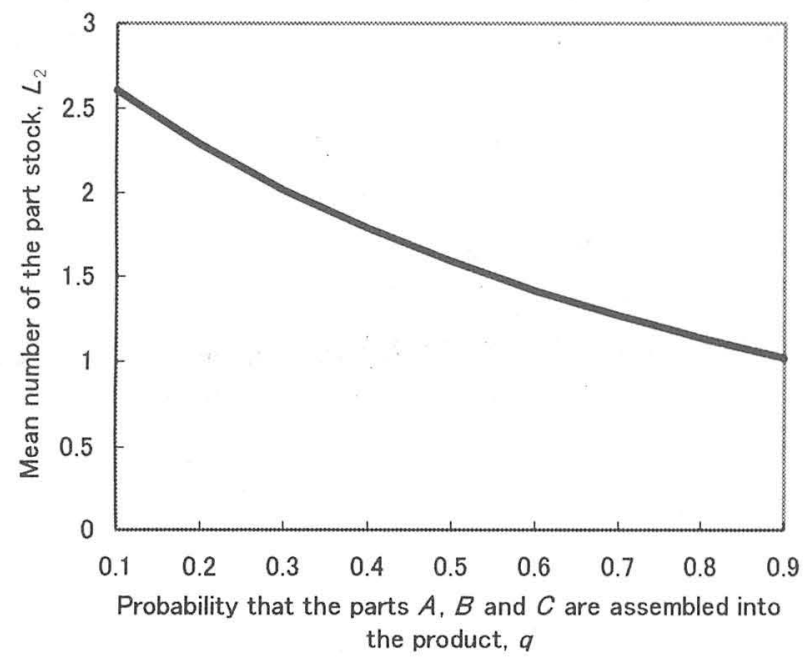

Fig.4 $q$ vs. $L_{2}\left(p_{1}=0.8, p_{2}=0.7, p_{3}=0.6\right)$

\section{Conclusion}

In this study, we treated two merge-type transfer line systems. The first system processes the merge-type transfer line system of three parallel. It is a manufacturing process of three kinds of part. The system assembled in parallel and completed at the same time. Next, the second system is the merge-type transfer line system with two stages in which three kinds of parts completed in the first stage. The second system conveyed to the following stage in series. The system has three kinds of part, which are supplied the next stage when the parts are completed in parallel and are gathered at the same time. We analyzed these systems by the Markov process at discrete times. Examples of numerical calculations were given for the obtained results. These treatments are useful for evaluating the characteristic quantities and performance in the design, control and operation of the systems.

Further, it is possible to analyze the merge-type transfer line systems in which there are processes to make four or more kinds of parts by the same method.

\section{References}

[1] Hitomi, K., Manufacturing Systems Engineering, 2nd edition, Kyouritsu-shupan, 63-65 (in Japanese) (2000).

[2] Hirakawa,Operations Management,Morikita-shuppan,

65 -70(in Japanese) (2000).

[3] Yamashiro, M., T. Takagi, Y. Kuba, $5^{\text {th }}$ International Conference on Engineering Design and Automation (2001).

[4] Gershwin, S. B., Manufacturing Systems Engineering, Prentice-Hall Inc. 19-32 (2000).

[5] Cox, D. R., H. D. Miller, The Theory of Stochastic Processes, Chapman and Hall, 43-46 (1965).

[6] Shamblin, J. E., G. T. Stevens, Jr, Operations Research, McGraw-Hill, Inc., 212-215(1974).

[7] Zalcom, M., P. Bartfai, J. Tomko, Point Processes and Queuing Problems, North-Holland Publishing Company, 413-426(1981). 